\title{
EL PAPEL DEL BOSQUE EN LOS PROCESOS HIDROLÓGICOS. EJEMPLOS EN LAS CUENCAS DE INVESTIGACIÓN DE VALLCEBRE (PREPIRINEO CATALÁN)
}

\author{
P. LLORENS ${ }^{(1)}$, R. POYATOS ${ }^{(1)}$, C. RUBIO(1), J. LATRON ${ }^{(2)}$ y F. GALLART ${ }^{(1)}$ \\ (1) Institut de Ciències de la Terra 'Jaume Almera' \\ Consejo Superior de Investigaciones Científicas \\ Solé i Sabarís, S/N. 08028 Barcelona \\ (2) Instituto Pirenaico de Ecología \\ Consejo Superior de Investigaciones Científicas \\ Avda. Montañana, 1005 . 50059 Zaragoza \\ Correo electrónico de contacto: pllorens@ija.csic.es
}

RESUMEN: Las cuencas de Vallcebre son representativas de áreas de montaña Mediterránea con forestación espontánea por pino albar (Pinus sylvestris L.) consecuencia del abandono de las labores agrícolas desde mediados del siglo XX. En este contexto, los procesos hidrológicos se han visto modificados como consecuencia de la aparición de un mosaico de cubiertas vegetales, formado por manchas del bosque original de robles (Quercus pubescens Willd.) o de pino albar dispersas en las terrazas de cultivo abandonadas, estas últimas, cubiertas mayoritariamente por prados mesófilos. Los flujos hídricos y compartimentos a escala de cada una de estas cubiertas vegetales están siendo estudiados en las cuencas de investigación de Vallcebre con la doble finalidad, comprender el funcionamiento de los procesos descriptores del uso de agua por diferentes cubiertas vegetales en áreas con importantes déficits hídricos y extrapolar estos conocimientos a escala de cuenca a partir de la modelización hidrológica.

ABSTRACT: The Vallcebre research catchments are representative of Mediterranean mountain areas with spontaneous afforestation by Scots pine (Pinus sylvestris L.) as a consequence of the abandonment of agricultural practices since the middle of the $X X$ century. In this context, hydrological processes have been modified as a consequence of the emergence of a mosaic of different land covers, formed by patches of the original oak forest (Quercus pubescens Willd.) or of Scots pine forest scattered in the old abandoned terraces mainly covered by mesophilous meadows. Water fluxes and stores have been studied at the Vallcebre research catchments with a twofold objective, the understanding of the processes describing the water use by different vegetation covers in areas with larger water deficits and the extrapolation of this knowledge at catchment scale by means of hydrological modelling. 
Palabras clave: hidrología, cuenca de investigación, bosque, Pre-Pirineo.

Key words: hydrology, experimental catchment, forest, Pre-Pyrenees.

Enviado el 22 de junio de 2005

Aceptado el 20 de septiembre de 2005

\section{Introducción}

Las áreas de montaña Mediterránea son fuentes de agua necesaria para el consumo de las zonas más pobladas situadas aguas abajo. Estas áreas participan de procesos hidrológicos muy diversos, que van de los descritos en medios húmedos hasta los característicos de medios secos, determinados por la propia estacionalidad del clima Mediterráneo. Por estos motivos el estudio del funcionamiento hidrológico de dichas áreas es de interés tanto fundamental como aplicado, y puede ayudar a la comprensión de las consecuencias del cambio global en los recursos hídricos.

Las áreas de Montaña mediterránea han sufrido importantes cambios en los usos del suelo a lo largo del siglo XX, principalmente debido a la forestación espontánea, mayoritariamente por pinares, de los antiguos campos de cultivo (Garcia Ruiz et al., 1996). Las consecuencias hidrológicas de estos cambios de uso han sido ampliamente demostradas a escala de cuenca (p. ej. Bosch y Hewlett, 1982; Sahin y Hall, 1996) y concretamente en cuencas de cabecera Pirenaicas (Gallart y Llorens, 2004).

De todos modos, es necesaria más información sobre los procesos que describen los usos del agua por diferentes cubiertas vegetales, especialmente en áreas con importante déficit hídrico, como es el caso de la montaña Mediterránea.

Las cuencas de investigación de Vallcebre fueron instrumentadas en 1989 con la finalidad de estudiar los procesos hidrológicos, de erosión y transporte de sedimentos en estas zonas de montaña fuertemente modificadas por las actividades agrícolas en el pasado y posteriormente abandonadas. De los primeros estudios realizados (Llorens, 1991) se desprendió el interés de analizar las consecuencias hidrológicas de la reforestación espontánea de los antiguos campos de cultivos a partir del estudio detallado de los procesos hidrológicos de diversas cubiertas vegetales.

Las investigaciones sobre los procesos hidrológicos que se llevan a cabo en estas cuencas pretenden ser útiles a largo plazo siguiendo los criterios establecidos por la Década Hidrológica Internacional 1965-1975 (Toebes y Ouryvaev, 1970), renovados recientemente en la Década sobre Predicciones en Cuencas No Aforadas de la I.A.H.S. 2003-2012 (Sivapalan et al., 2003).

Este trabajo presenta una síntesis de los resultados de los estudios sobre el papel del bosque en los procesos hidrológicos realizados en las cuencas de investigación de Vallcebre durante 15 años (1989-2004). 


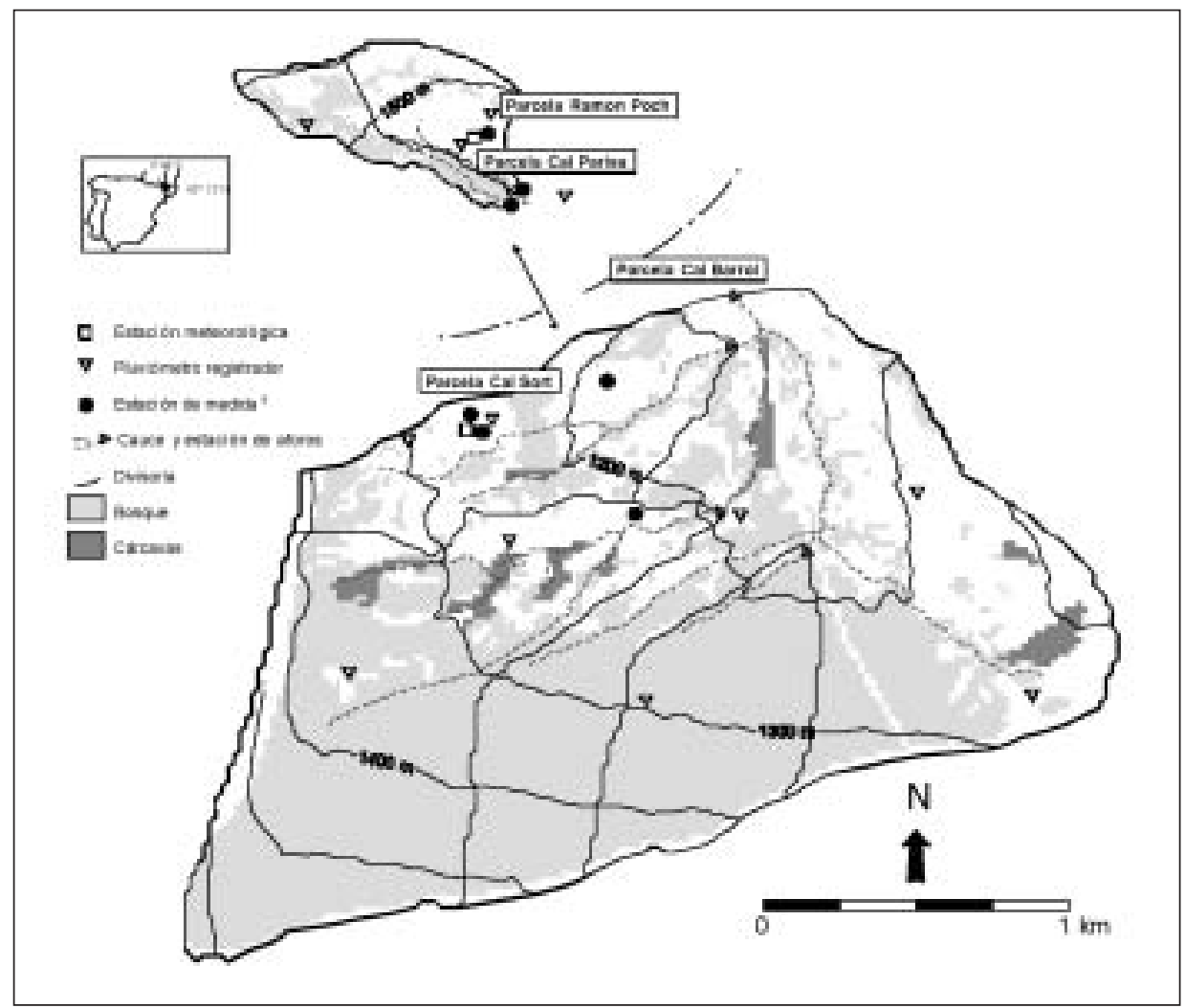

Figura 1. Mapa de situación de las cuencas de investigación de Vallcebre, mostrando el diseño instrumental general de las cuencas y la situación de parcelas de medición de flujos en diferentes cubiertas vegetales.

\section{El área de estudio}

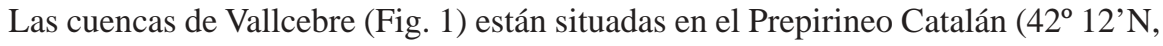
$1^{\circ} 49^{\prime} \mathrm{E}$ ), a una altitud entre 1100 y $1700 \mathrm{~m}$. La zona de estudio comprende dos grupos de cuencas separados aproximadamente $1.5 \mathrm{~km}$. El grupo principal lo forma la Cuenca de Cal Rodó (4.17 km²), que engloba 3 subcuencas (Ca l'Isard, Can Vila and Sta. Magdalena), mientras que el grupo pequeño se denomina Cal Parisa y consiste en un par de cuenca de tamaño similar (unos $0.16 \mathrm{~km}^{2}$ cada una).

El clima de la zona es Submediterráneo, con una temperatura media anual de $7.3^{\circ}$ C, una precipitación media anual de $924 \mathrm{~mm}$ y una evapotranspiración de referencia de unos 700mm (Gallart et al., 2002).

El susbstrato geológico lo forman arcillas, margas y calizas continentales del Paleoceno (facies Garumniense). 
Los suelos sobre substrato arcilloso son de textura franco-limosa, con un contenido de la fracción limo superior al $60 \%$ y han sido notablemente modificados por el aterrazamiento. Los elevados contenidos de las fracciones finas (limo y arcilla) y de materia orgánica (próximos al 8\%), confieren a estos suelos una elevada capacidad de retención hídrica, con valores promedio para la curva característica de la humedad de 0.60, 0.35 y $0.20 \mathrm{~cm}^{3} \cdot \mathrm{cm}^{-3}$ a saturación, capacidad de campo y punto de marchitamiento permanente respectivamente (Rubio y Llorens, 2003; 2004).

La vegetación climácica es el bosque de Quercus pubescens Willd. y de Pinus sylvestris L. en las zonas umbrías o más frías. La mayor parte de las vertientes de pendiente relativamente suave fueron deforestadas, aterrazadas y utilizadas para la agricultura hasta mediados del siglo XX. Actualmente, las terrazas de cultivo abandonadas están cubiertas por prados mesófilos, estas terrazas están siendo progresivamente colonizadas por Pinus sylvestris (en la actualidad cubren un $30 \%$ de las terrazas). Las áreas más marginales, y por tanto abandonadas precedentemente, tienen cubiertas forestales bastante densas, mientras que en las áreas abandonadas tardíamente predominan los árboles dispersos o agrupados en pequeñas manchas (Poyatos et al., 2003).

Un análisis de los cambios de la vegetación en los últimos 40 años indica que en 1957, los bosques (de diversas densidades) cubrían un 39\% de la cuenca, mientras que en 1996 representaban un 64\% (Poyatos et al. 2003).

\section{Diseño instrumental}

\subsection{Instrumentación permanente}

La instrumentación de las cuencas de investigación de Vallcebre se inició en 1989 (Llorens y Gallart, 1992; Balasch et al., 1992). Además de la monitorización de la meteorología, la precipitación y los caudales, diversas variables de estado (humedad del suelo, tensiometría y niveles piezométricos) y procesos (interceptación y transpiración de diversas cubiertas vegetales) son medidos permanentemente o lo han sido durante periodos de varios años (Gallart et al., en prensa c).

La red pluviométrica consiste en 12 pluviómetros registradores (tabla 1), conectados a colectores de datos registrando, en la mayoría de los dispositivos, el momento en que se produce el vuelco del balancín $(0.2 \mathrm{~mm})$, con una precisión de $1 \mathrm{seg}$.

Dos estaciones meteorológicas instaladas en cada uno de los grupos de cuencas (Cal Rodó y Cal Parisa) registran la temperatura y humedad relativa del aire, la radiación global y neta, así como la velocidad y dirección del viento.

Todas las cuencas disponen de una estación de aforos provista de una estructura de control, un sensor de nivel y uno de temperatura del agua, que registran los datos a intervalos variables entre 2 y 20 minutos. Las estaciones de las cuencas de Cal Rodó, Ca l'Isard y Can Vila (tabla 1) están provistas de muestreadores automáticos de agua y sedimento en suspensión, así como de sensores de turbidez por retrodifusión de radiación 
Tabla 1. Características y instrumentación permanente de las Cuencas de Vallcebre (adaptado de Latron 2003; Gallart et al., en prensa c).

\begin{tabular}{|c|c|c|c|c|c|c|}
\hline & $\begin{array}{c}\text { Cal } \\
\text { Parisa } 1\end{array}$ & $\begin{array}{c}\text { Cal } \\
\text { Parisa } 2\end{array}$ & $\begin{array}{l}\text { Cal } \\
\text { Rodó }\end{array}$ & $\begin{array}{c}\mathrm{Ca} \\
\text { l'Isard }^{\mathrm{s}}\end{array}$ & $\begin{array}{l}\text { Can } \\
\text { Vila }^{\mathrm{s}}\end{array}$ & $\begin{array}{c}\text { Sta. } \\
\text { Magdalena }^{\mathrm{s}}\end{array}$ \\
\hline Area $\left(\mathbf{K m}^{2}\right)$ & 0.17 & 0.13 & 4.17 & 1.32 & 0.56 & 0.53 \\
\hline $\begin{array}{l}\text { Substrato }(\%) \\
\text { Calizas } \\
\text { Margas }\end{array}$ & $\begin{array}{l}11.3 \\
88.7\end{array}$ & $\begin{array}{r}7.4 \\
92.6\end{array}$ & $\begin{array}{l}49.6 \\
50.4\end{array}$ & $\begin{array}{l}54.1 \\
45.9\end{array}$ & $\begin{array}{r}3.1 \\
96.9\end{array}$ & $\begin{array}{r}100.0 \\
0.0\end{array}$ \\
\hline Bosque (\%) & 13.5 & 60.5 & 60.1 & 70.3 & 33.7 & 95.8 \\
\hline $\begin{array}{l}\text { Pluviometría } \\
\text { P }\end{array}$ & $\begin{array}{l}1989- \\
3(1)\end{array}$ & $\begin{array}{c}1989-2000 \\
1\end{array}$ & $\begin{array}{c}1994- \\
3\end{array}$ & $\begin{array}{c}1994- \\
2\end{array}$ & $\begin{array}{c}1994- \\
2\end{array}$ & $\begin{array}{c}1994- \\
1\end{array}$ \\
\hline $\begin{array}{l}\text { Meteorología } \\
\text { T } \\
\text { HR } \\
\text { Rg } \\
\text { Rn } \\
\text { V y D }\end{array}$ & $\begin{array}{c}1989-2003 \\
1 \\
1 \\
1 \\
1 \\
1\end{array}$ & $\begin{array}{l}- \\
- \\
- \\
- \\
- \\
-\end{array}$ & $\begin{array}{l}- \\
- \\
- \\
- \\
-\end{array}$ & $\begin{array}{l}- \\
- \\
- \\
- \\
- \\
-\end{array}$ & $\begin{array}{c}1996- \\
1 \\
1 \\
1 \\
1 \\
1\end{array}$ & $\begin{array}{l}- \\
- \\
- \\
- \\
- \\
-\end{array}$ \\
\hline $\begin{array}{l}\text { Estaciones de aforo } \\
\text { Q } \\
\text { Tq } \\
\text { Turbidez } \\
\text { Muestreadores }\end{array}$ & $\begin{array}{c}1989-2003 \\
1 \\
1 \\
- \\
1\end{array}$ & $\begin{array}{c}1991-2003 \\
1 \\
1 \\
- \\
-\end{array}$ & $\begin{array}{c}1991- \\
1 \\
1 \\
2 \\
2\end{array}$ & $\begin{array}{c}1991- \\
1 \\
1 \\
2 \\
1\end{array}$ & $\begin{array}{c}1995- \\
1 \\
1 \\
1 \\
1\end{array}$ & $\begin{array}{c}1995-2001 \\
1 \\
1 \\
- \\
-\end{array}$ \\
\hline $\begin{array}{l}\text { Humedad del suelo } \\
\text { TDR } \\
\text { Tensiometría }\end{array}$ & $\begin{array}{l}1993- \\
7 \\
2\end{array}$ & - & $\begin{array}{l}- \\
-\end{array}$ & $\begin{array}{l}- \\
-\end{array}$ & $\begin{array}{l}1996- \\
2 \\
1\end{array}$ & - \\
\hline Piezometría & $\begin{array}{c}1989- \\
1^{\mathrm{m}}\end{array}$ & - & $\begin{array}{c}1996- \\
3^{\mathrm{a}}\end{array}$ & - & $\begin{array}{c}1996- \\
1^{\mathrm{a}}\end{array}$ & - \\
\hline \multicolumn{7}{|c|}{$\begin{array}{l}\text { Subcuencas de Cal Rodó; }{ }^{\mathrm{m}} \text { medidas manuales en pozos; }{ }^{a} \text { piezómetros automáticos. } \mathrm{P}=\text { plu- } \\
\text { viómetro registrador; } \mathrm{T}=\text { temperatura del aire; } \mathrm{HR}=\text { humedad relativa del aire; } \mathrm{Rg}=\text { radiación } \\
\text { global; } \mathrm{Rn}=\text { radiación neta; } \mathrm{V} \text { y } \mathrm{D}=\text { =velocidad y dirección del viento; } \mathrm{Q}=\text { caudal; } \mathrm{Tq}=\text { temperatu- } \\
\text { ra del agua. }\end{array}$} \\
\hline
\end{tabular}

infrarroja y, en las dos primeras, de un sensor de sólidos en suspensión por atenuación de haz de ultrasonidos (Gallart et al., 2005 a y b).

La humedad del suelo se mide en las cuencas de Vallcebre desde 1993 (Rabadà, 1995) utilizando el método de la Reflectometría en el dominio temporal (TDR) en 9 perfiles situados en las principales unidades geoecológicas ( 7 en la cuenca de Cal Parisa y 2 en la cuenca de Can Vila). Estos perfiles consisten en conjuntos de 4 sondas de TDR de $20 \mathrm{~cm}$ instaladas verticalmente en el suelo a profundidades de 0-20, 20-40, 40-60 y 
60-80 cm. Estos sensores son leídos con periodicidad semanal mediante un comprobador de cables Tektronix 1502-C (tabla 1).

La red de tensiometría, instalada a finales de 1995, consta de tensiómetros en continuo (lecturas cada 20 minutos) instalados a 20, 40 y $60 \mathrm{~cm}$.

Las medidas piezométricas se realizan semanalmente en 4 pozos abandonados. Además, a finales de 1995, se instrumentaron 4 piezómetros registradores (con lecturas cada 20 minutos) en la cuenca de Cal Rodó.

3.2. Instrumentación semi-permanente para la medida de los flujos de agua de diferentes cubiertas vegetales

\section{Parcela de Pino albar Ramon Poch y parcela de prado mesófilo Cal Parisa}

En la cuenca de Cal Parisa se ha monitorizado durante 10 años (1993-2002) una parcela experimental con una cubierta monoespecífica de Pinus sylvestris jóvenes y sin sotobosque, con la finalidad de evaluar el balance hídrico de una parcela representativa de la forestación espontánea. La parcela fue instrumentada para la medición en continuo de la interceptación del agua de lluvia (Llorens et al., 1997) y la transpiración del pinar (Oliveras y Llorens, 2001), además disponía de un registro en continuo de tensiometría y semanal de humedad del suelo. La interceptación forestal se midió con un colector de $1 \mathrm{~m}^{2}$ para la recolección de la precipitación incidente, 9 colectores de trascolación de $1 \mathrm{~m}^{2}$ cada uno agrupados en tres grupos, así como 7 anillos colectores de escorrentía cortical, también agrupados en tres conjuntos. Cada grupo disponía de un dispositivo tipo balancín conectado a un colector de datos que almacenaba la información cada 5 minutos (Llorens et al., 1997). La instrumentación para la medida del flujo de savia constaba de 6 sondas de calentamiento constante (Granier, 1985, 1987) conectadas al mismo colector de datos con un registro cada 20 minutos (tabla 2), más 6 sondas suplementarias durante un experimento de validación del método para uso prolongado (Oliveras y Llorens, 2001).

Durante el mismo período se registró en esta cuenca la temperatura y humedad del aire con 2 aspiro-psicrómetros a dos alturas distintas sobre una terraza abandonada así como los términos del balance energético (radiación neta y flujo de calor en el suelo) (Tabla 2), permitiendo la estimación de la evapotranspiración real de un prado mesófilo por el método combinado del balance energético y la relación de Bowen (Poyatos y Llorens, 2003).

\section{Parcela de Pino albar Cal Sort y parcela de robles Cal Barrol}

Desde el 2003 se dispone de 2 nuevas estaciones de medición de la transpiración forestal, Cal Sort con una cubierta de Pinus sylvestris Cal Barrol con una cubierta de Quercus pubescens. La parcela de pino albar está situada sobre una antigua terraza de cultivo y tiene una cubierta monoespecífica con pies jóvenes y un sotobosque muy escaso (Buxus sempervirens L.). En la parcela de robles, en cambio, coexisten otras especies forestales (Prunus avium L. y Fraxinus excelsior L.), así como un denso sotobos- 
Tabla 2. Características e instrumentación de las parcelas de estudio de la (evapo)transpiración y interceptación forestal (Adaptado de Llorens et al., 1997; Oliveras y Llorens, 2001;

Poyatos et al., 2005).

\begin{tabular}{|c|c|c|c|c|}
\hline \multirow[b]{2}{*}{ Cubierta } & \multicolumn{2}{|c|}{ Cal Parisa } & \multirow{2}{*}{$\begin{array}{c}\text { Cal Sort } \\
\text { P. sylvestris }\end{array}$} & \multirow{2}{*}{$\begin{array}{c}\text { Cal Barrol } \\
\text { Q. pubescens }\end{array}$} \\
\hline & Prado mesófilo & P. sylvestris & & \\
\hline $\begin{array}{l}\text { Parámetros } \\
\text { Densidad }(\text { pies ha } \\
\text { DBH }(\mathrm{cm}) \\
\text { Area basal }\left(\mathrm{m} 2 ~ h a^{-1}\right) \\
\text { Altura }(\mathrm{m})\end{array}$ & $\begin{array}{l}- \\
- \\
- \\
0.4\end{array}$ & $\begin{array}{r}2400 \\
17.3 \\
10.0\end{array}$ & $\begin{array}{r}2165 \\
15.2 \\
44.7 \\
11.0\end{array}$ & $\begin{array}{l}828 \\
21.1 \\
32.5 \\
10.6\end{array}$ \\
\hline $\begin{array}{l}\text { Meteorología } \\
\text { T } \\
\text { HR } \\
\operatorname{Rg} \\
\text { Rn } \\
\text { V y D }\end{array}$ & $\begin{array}{l}1 \\
1 \\
1 \\
1 \\
1\end{array}$ & $\begin{array}{l}- \\
-\end{array}$ & $\begin{array}{c}2003^{t}- \\
1 \\
1 \\
1 \\
1\end{array}$ & $\begin{array}{c}2003^{t}- \\
1 \\
1 \\
1 \\
1\end{array}$ \\
\hline $\begin{array}{l}\text { Interceptación } \\
\text { Precipitación } \\
\text { Canales }\left(\mathrm{m}^{2}\right) \\
\text { Anillos escorrentía }\end{array}$ & & $\begin{array}{c}1993-2002 \\
1 \\
9 \\
7\end{array}$ & $\begin{array}{l}- \\
-\end{array}$ & $\begin{array}{c}2004- \\
1 \\
6 \\
0\end{array}$ \\
\hline $\begin{array}{l}\text { Transpiración } \\
\text { BREB } \\
\text { Sensores flujo savia }\end{array}$ & $\begin{array}{c}1993-2002 \\
1 \\
-\end{array}$ & $\begin{array}{c}1993-2000 \\
- \\
6(+6)\end{array}$ & $\begin{array}{c}2003- \\
- \\
12\end{array}$ & $\begin{array}{c}2003- \\
- \\
12\end{array}$ \\
\hline $\begin{array}{l}\text { Humedad del suelo } \\
\text { TDR } \\
\text { Tensiometría }\end{array}$ & $\begin{array}{c}1993- \\
1 \\
1\end{array}$ & $\begin{array}{c}1993- \\
1 \\
1\end{array}$ & $\begin{array}{l}2003- \\
2^{\mathrm{a}} \\
-\end{array}$ & $\begin{array}{l}2003- \\
2^{\mathrm{a}} \\
-\end{array}$ \\
\hline \multicolumn{5}{|c|}{$\begin{array}{l}\text { Torres meteorológicas ( } 2 \mathrm{~m} \text { sobre las cubiertas de bosque); }{ }^{\text {a }} \text { uno de los perfiles es de registro } \\
\text { continuo. } \\
\mathrm{T}=\text { temperatura del aire; } \mathrm{HR}=\text { humedad relativa del aire; } \mathrm{Rg}=\text { radiación global; } \mathrm{Rn}=\text { radiación } \\
\text { neta; } \mathrm{V} \text { y } \mathrm{D}=\text { velocidad y dirección del viento; } \mathrm{BREB}=\text { Estación combinada de Balance energé- } \\
\text { tico y Razón de Bowen. }\end{array}$} \\
\hline
\end{tabular}

que principalmente de B.sempervirens. En ambas parcelas se mide la transpiración con 12 sensores de flujo de savia de tipo Granier conectados a un colector de datos que registra la inforamación cada 10 minutos. Además, cada una de ellas dispone de una torre situada aproximadamente a $2 \mathrm{~m}$ sobre las copas (Tabla 2) con el registro en continuo de la principales variables meteorológicas, y unos perfiles de medición de la humedad del suelo por el método TDR (Poyatos et al., 2005). Otras medidas complementarias en estas parcelas incluyen la intercomparación de tres metodologías de medida del flujo de savia en Q.pubescens, la evaluación de la vulnerabilidad al embolismo por sequía en el xilema de P.sylvestris y el seguimiento en ambas especies del potencial hídrico de base y al mediodía. 


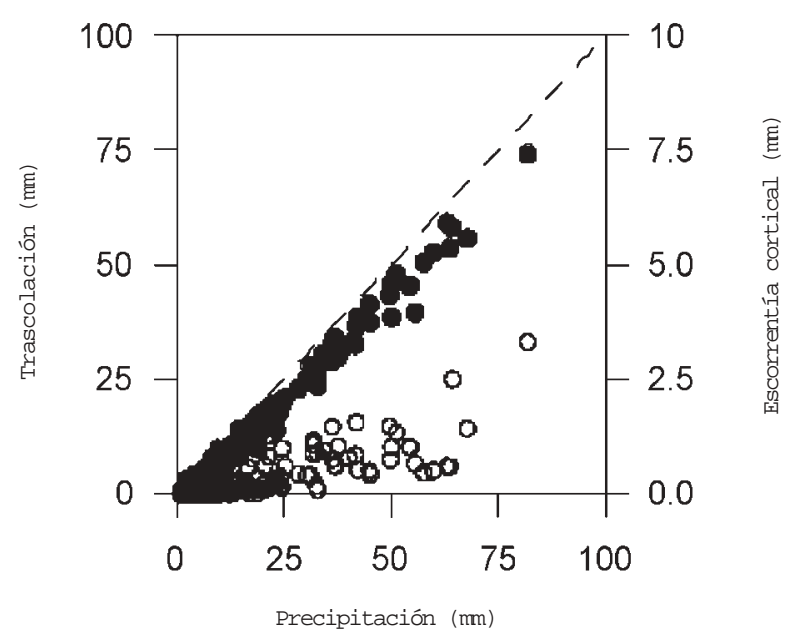

Figura 2. Relaciones entre la precipitación (fuera del bosque) a escala de evento y la trascolación (círculos rellenos) y la escorrentía cortical (círculos vacíos) en la parcela R. Poch de Cal Parisa.

En la parcela de robles se inició en el 2004 la instalación de seis colectores de trascolación de $1 \mathrm{~m}^{2}$ para medir la interceptación.

\section{Principales resultados}

\subsection{Interceptación forestal}

La trascolación media de la parcela experimental de pino albar Ramon Poch en Cal Parisa (julio 1993-Agosto 2000) representa un 74\% de la precipitación, mientras que la escorrentía cortical supone solo un 2\% (Figura 2), suponiendo una tasa de interceptación anual del 24\% (Llorens et al., 2003b).

El análisis del papel de las condiciones meteorológicas en la interceptación del agua de lluvia a escala de evento, realizado por Llorens et al. (1997), permitió identificar tres tipos de eventos (Tabla 3): Eventos de las mismas características meteorológicas que los descritos en condiciones templado-húmedas, estos eventos se producen a lo largo de todo el año y representan la mayor parte de la precipitación (62\%) y de la interceptación (57\%) anuales (clase I). Eventos originados por tormentas de elevada intensidad con características propias del clima mediterráneo (tormentas convectivas de verano), que producen interceptaciones relativas moderadas y que representan un $24 \%$ de la precipitación anual (clase II). Por último, eventos también de tipo mediterráneo con interceptaciones relativas muy elevadas debido a unas condiciones atmosféricas muy 
Tabla 3. Clasificación de los eventos lluviosos en función de su duración, intensidad y condiciones atmosféricas y tasas de interceptación resultantes

(Adaptado de Llorens et al., 1997).

\begin{tabular}{|l|c|c|c|}
\hline & Clase I & Clase II & Clase III \\
\hline Duración (h) & $\begin{array}{c}26 \\
\text { largos }\end{array}$ & $\begin{array}{c}5 \\
\text { cortos }\end{array}$ & $\begin{array}{c}12 \\
\text { medios }\end{array}$ \\
\hline Intensidad precipitación (mm h') & $\begin{array}{c}1.6 \\
\text { baja }\end{array}$ & $\begin{array}{c}7.6 \\
\text { alta }\end{array}$ & $\begin{array}{c}1.2 \\
\text { baja }\end{array}$ \\
\hline Humedad atmosférica: & 0.3 & 1.1 & 2.0 \\
Déficit de saturación (mb) & $\begin{array}{c}\text { Próxima } \\
\text { a la saturación }\end{array}$ & Baja & Muy baja \\
\hline Interceptación (\%) & & & \\
Relativa & 15 & 13 & 49 \\
Contribución Interceptación anual & 57 & 24 & 23 \\
Porcentaje de precipitación anual & 62 & 18 & 8 \\
\hline
\end{tabular}

secas y que aún representando una parte muy poco importante de la precipitación total $(8 \%)$ tienen una contribución relevante a la interceptación anual (23\%).

Los valores de interceptación relativa resultan muy similares de una estación a otra ya que se produce un efecto de compensación entre la intensidad de precipitación y las condiciones atmosféricas a lo largo del año debido a la distribución de los tres tipos de eventos (Gallart et al., 2002).

Los resultados de la interceptación en la parcela de robles Cal Barrol son aún muy preliminares para ser presentados en este trabajo.

\subsection{Transpiración}

Las observaciones realizadas sobre los 3 tipos de vegetación estudiados (Llorens et al., 2003c, Oliveras y Llorens, 2001; Poyatos y Llorens, 2003; Poyatos et al., 2005) han permitido observar la dependencia de las tasas de transpiración respecto a las variables atmosféricas y la humedad del suelo para cada tipo de cubierta.

Las medidas de evapotranspiración real realizada en la parcela de pasto mesófilo Cal Parisa indican que durante el período vegetativo (con temperatura del aire superior a $7^{\circ} \mathrm{C}$ ) la transpiración relativa (cociente entre la evapotranspiración real y la de referencia) es próxima a la unidad cuando el déficit de humedad del suelo (Soil Moisture Deficit, SMD) es inferior a 0.8, es decir humedades del suelo un $20 \%$ superiores a la humedad del suelo mínima registrada (Granier y Loustau, 1994), mientras que disminuye hasta valores próximos al 0.4 cuando el déficit es elevado (Fig. 3). Durante la esta- 


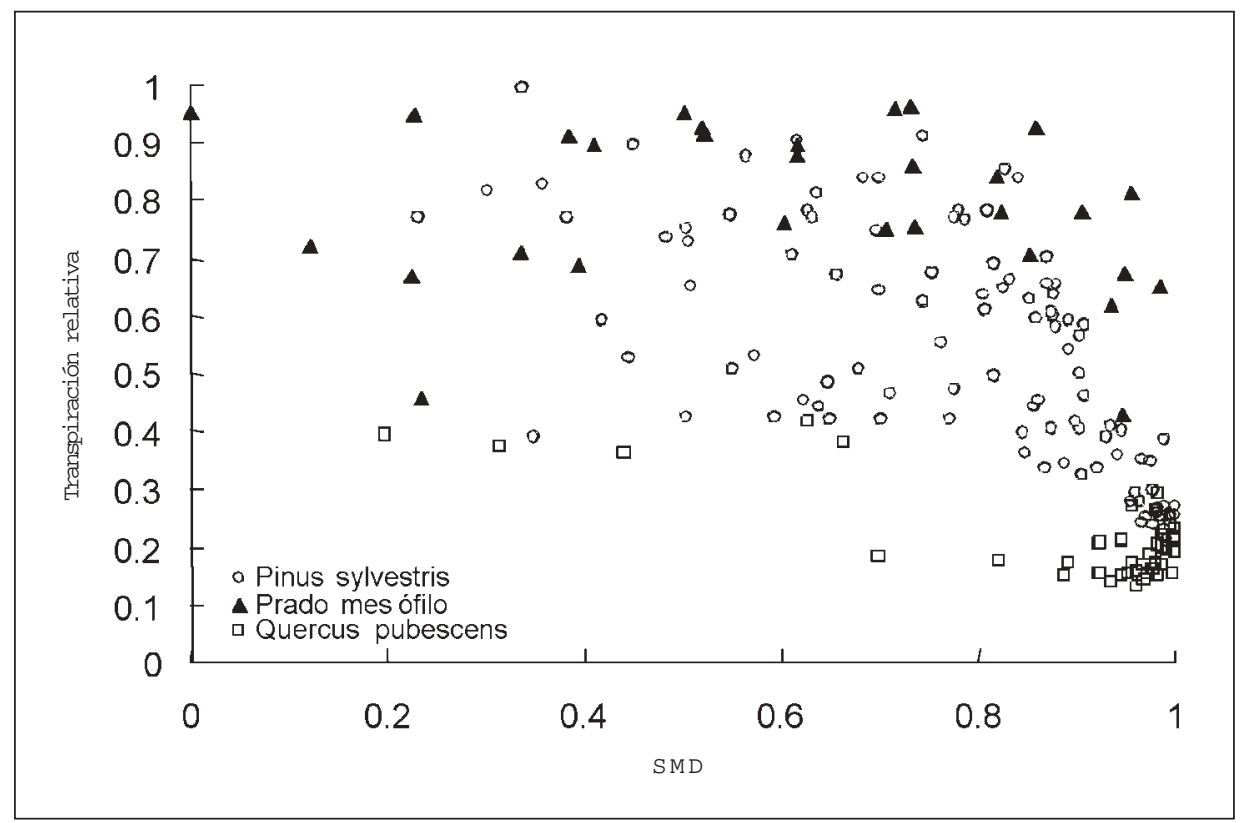

Figura 3. Valores de transpiración relativa a la evapotranspiración de referencia (T/ET $)$ en función del déficit hídrico del suelo (SMD). Los valores del prado mesófilo se obtuvieron entre 1994 y 1999, los de Q.pubescens, durante 2003 y los de P. Sylvestris durante 2003 y 2004.

ción fría, aunque exista disponibilidad hídrica, la evapotranspiración relativa es baja (alrededor del 30\%) debido a la senescencia de los pastos, y aumenta progresivamente al comenzar de nuevo el periodo de actividad vegetal a finales de la primavera (Poyatos y Llorens, 2003).

En la Parcela Ramon Poch (P. sylvestris) el principal resultado obtenido fue la demostración de la validez del método de medida del flujo de savia por disipación térmica (Granier, 1985; 1987) para la monitorización en continuo de la transpiración en la especie Pinus durante periodos largos de tiempo, es decir no solo para un único periodo vegetativo tal y como se consideraba hasta el momento (Oliveras y Llorens, 2001).

La transpiración en las parcelas forestales, Cal Sort y Cal Barrol, que se están estudiando en la actualidad (tabla 2) muestran importantes diferencias entre especies y entre periodos vegetativos secos y húmedos para una misma especie (Poyatos et al., 2005).

Durante periodos vegetativos sin condiciones de stress importantes, en los cuales un $75 \%$ o más de los días tienen un déficit de saturación del suelo en los 30 primeros centímetros (Soil Moisture Deficit, SMD) inferior al 0.8, la transpiración de los pinos (Pinus sylvestris) representa un 65\% de la evapotranspiración de referencia. Por el contrario, en condiciones de stress hídrico importante, cuando un $40 \%$ o más de los días tie- 
nen un SMD superior al 0.8, la transpiración de los pinares desciende hasta el $45 \%$ de la evapotranspiración de referencia (Poyatos et al., 2005). Es de destacar que la frecuencia de días con moderado déficit hídrico (SMD>0.6) es similar, independientemente de si es un verano seco (2003) o húmedo (2004), indicando un uso intensivo por parte de la vegetación de la reserva hídrica más superficial.

La transpiración de los robles (Quercus pubescens) representa menos de la mitad de la de los pinos (un 19\% de la evapotranspiración de referencia) para un período vegetativo extremadamente seco (2003). De todos modos, el denso sotobosque bajo el robledal, no estudiado hasta el momento, contribuye sin duda significativamente a la transpiración total a escala de parcela, tal como indican trabajos realizados sobre cubiertas similares (p. ej. Cermák et al., 1998).

\subsection{Contenido de agua en el suelo}

El análisis de los registros de humedad del suelo en Vallcebre (Gallart et al. 1997) mostró por un lado que los suelos bajo cubierta de árboles están generalmente más secos que en los prados (mesófilos) o campos abandonados, y por otro lado que la transferencia subsuperficial de agua, que alimenta las áreas frecuentemente saturadas ya sea a pie de vertiente o en las partes internas de las terrazas, se interrumpe durante los períodos secos. Llorens et al. (2003a) observaron que la variabilidad espacial de la humedad del suelo es mayor en condiciones intermedias de humedad (humedad volumétrica del 30$35 \%$ ) y decrece tanto en condiciones secas (20-25\%) como húmedas (40-45\%).

El análisis de la estabilidad temporal de la humedad del suelo en Vallcebre, realizado según el criterio definido por Vachaud et al., (1985) que permite determinar la persistencia de un modelo de comportamiento de cada perfil respecto a la media, nos muestra una ordenación de los perfiles de más secos a más húmedos (Fig. 4a). Las diferencias relativas, respecto a la media, son mayores en los perfiles sistemáticamente más húmedos (hasta un $50 \%$ en una terraza saturada), mientras que para los perfiles más secos las diferencias máximas son de $-20 \%$. Los tres perfiles más secos corresponden a perfiles bajo bosque, los perfiles en vertiente con cubierta herbácea tienen valores próximos a la media y los perfiles en terrazas tienden a ser más húmedos que la media (Llorens et al., 2003a).

La estacionalidad de los contenidos de humedad del suelo en 3 perfiles representativos: un terraza frecuentemente saturada, situada a pie de vertiente, y dos perfiles situados en la misma vertiente, en una situación intermedia, uno bajo bosque (Parcela Ramon Poch) y el otro cubierto por un prado mesófilo, fue analizada por Gallart et al. (2002). La figura 4b, donde se representan las medias quincenales durante el período 1993-99, muestra dicha evolución estacional. El año se inicia con suelos húmedos, debido a que el otoño es la estación más húmeda. Durante el invierno los suelos a media vertiente (bosque y prado mesófilo) van secándose, aunque la demanda atmosférica no es muy elevada en este período y los aportes por precipitación son limitados (el invierno es la 

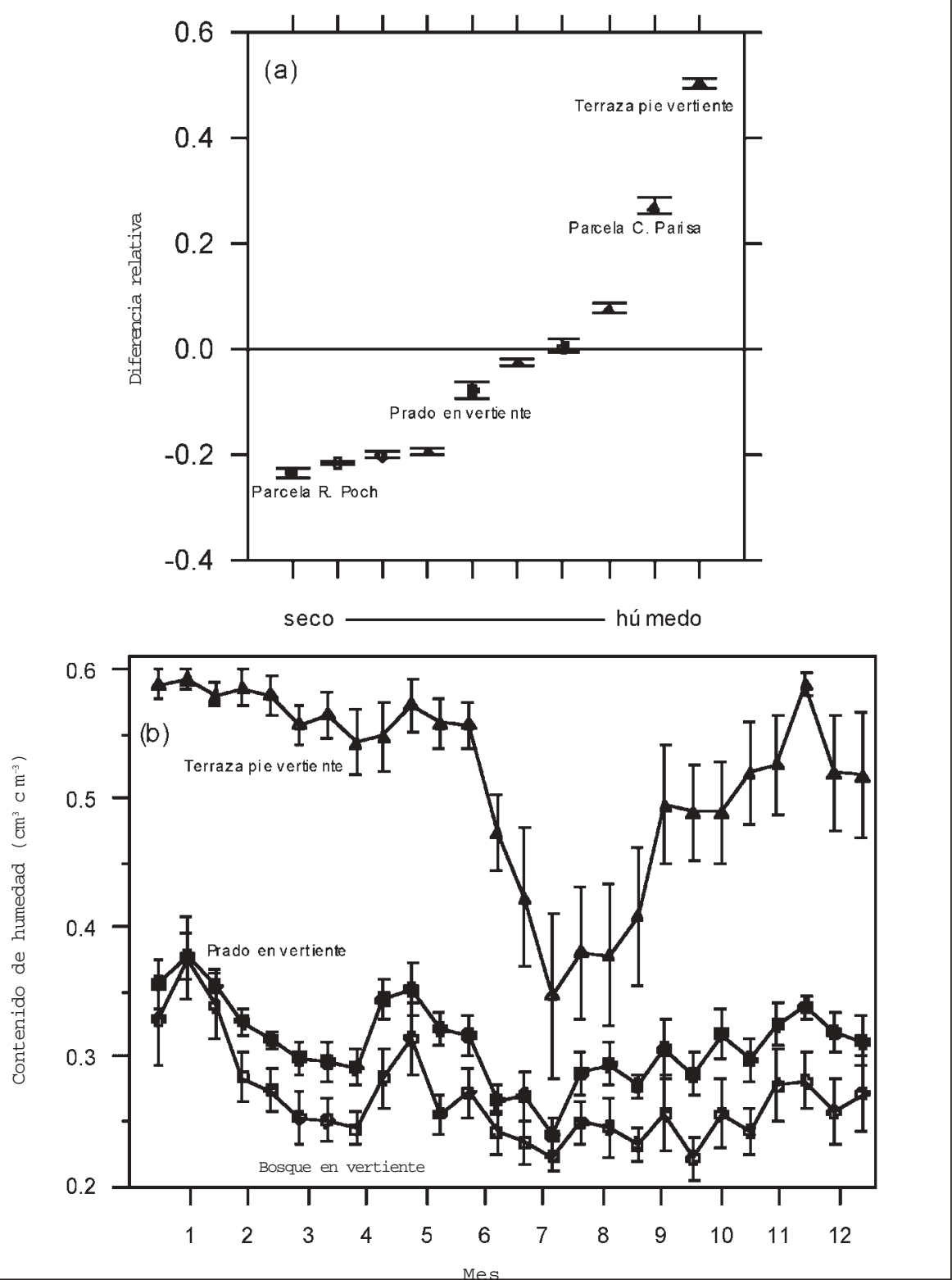

Figura 4. (a) Clasificación de los diferentes perfiles de humedad del suelo, de más seco a más húmedo, en función de la diferencia relativa entre le valor medio de cada perfil y el promedio de todos los perfiles. (b) Medias quincenales (1993-99) de la humedad volumétrica del suelo medida en 3 perfiles representativos.

En ambos gráficos, las barras representan los errores estándar; los triángulos terrazas con pastos mesófilos; los círculos rellenos vertientes con pastos mesófilos y los círculos vacíos vertientes con pinares. 
estación más seca en Vallcebre). En cambio, la terraza a pie de la vertiente mantiene la humedad próxima a la saturación. Al llegar la primavera, como consecuencia de las lluvias y una demanda atmosférica limitada, los suelos van recuperando la humedad. Es a partir del mes de Junio cuando la fuerte demanda evapotranspirativa ya no se ve compensada por las precipitaciones, y se inicia una desecación de los perfiles, que es más pronunciada en le perfil a pie de vertiente al producirse una interrupción de la transferencia subsuperficial de agua. A finales de Julio es cuando se alcanzan los niveles más bajos de humedad del suelo. Posteriormente, la llegada de las precipitaciones de Agosto y de los meses sucesivos y la reducción de la demanda evapotranspirativa provocan una recuperación de la humedad, primero en los perfiles a media vertiente y posteriormente se produce la saturación de la terraza a pie de vertiente.

Comparando los perfiles situados a media vertiente, se observa que el perfil situado en el bosque está siempre más seco que el del claro, siendo las diferencias mínimas en los momentos más húmedos o más secos. Después del verano, la recuperación de la humedad del perfil del claro es más rápida y acentuada que la del perfil en bosque.

\subsection{Modelización}

Interceptación. La interceptación de la lluvia por la cubierta de pinos fue simulada a escala de evento utilizando el modelo de Gash (1979) y tres alternativas distintas para la obtención de los parámetros necesarios. El ajuste del modelos empleados es similar al obtenido en clima templado para el mismo tipo de cubierta y por tanto suficientemente robusto para ser utilizado en condiciones de montaña Mediterránea (Llorens, 1997).

El análisis de sensibilidad realizado indica que el modelo es sensible a las características estructurales de la cubierta, y especialmente al parámetro que determina a la capacidad máxima de saturación de la cubierta (Llorens, 1997), por este motivo se desarrolló una metodología para la determinación experimental y de forma simplificada, de este parámetro (Llorens y Gallart, 2000).

Transpiración. La modelización de la transpiración a escala diaria se ha abordado en el caso de Pinus sylvestris, dada su importancia como agente colonizador de las antiguas terrazas de cultivo. Un modelo simple que calcula la transpiración diaria a partir de la evapotranspiración de referencia y el déficit de humedad del suelo (SMD) se ha parametrizado a partir de datos de 2003 y 2004. Este modelo captura tanto el cierre estomático debido a la sequedad atmosférica (Oren et al., 1999) como la reducción de la respuesta de la vegetación a la demanda evaporativa bajo condiciones de sequía edáfica (Granier y Loustau, 1994). A pesar de la correcta modelización de la dinámica de la transpiración, este modelo la sobreestima, especialmente en periodos secos (Poyatos et al., 2005).

Propiedades hídricas de los suelos. A partir de propiedades físico-químicas de los suelos de fácil determinación, se ha validado el Modelo Rosetta (Schaap et al., 2001) y 
desarrollado Funciones de Edafo-Transferencia específicas para los suelos de la cuenca de Can Vila con la finalidad de obtener los parámetros hidrodinámicos necesarios para la construcción de las curvas de succión (Rubio y Llorens, 2003, 2004).

Efecto del cambio de cubierta vegetal en la respuesta hidrológica. El balance de agua ha sido simulado en la cuenca de Can Vila utilizando el modelo SIMBAL de paso de tiempo diario, que considera explícitamente la interceptación y la transpiración por distintos tipos de cubiertas vegetales, con la finalidad de estudiar las consecuencias hidrológicas del cambio de cubierta vegetal (Llorens, 1993; Llorens et al., 2003b). El modelo ha sido validado con un registro de caudales de 3 años registrados en la cuenca de Can Vila con un buen ajuste tal como indica el valor de criterio de Nash de 0.88 (Nash y Sutcliffe, 1970), además se ha realizado una validación interna con la reserva de agua en el suelo estimada para toda la cuenca (Latron, 2003), observándose que el modelo ajusta con precisión las variaciones temporales de esta (Llorens et al., 2003b). Para la simulación del cambio de cubiertas del suelo se utilizó una serie de 10 años de precipitaciones medidas en Vallcebre y una serie de evapotranspiración potencial sintética calculada a partir de una serie más corta y se definieron varios escenarios combinando los 3 tipos de cubiertas representativas de la zona de estudio (prados mesófilos, pinares y robledales). Los resultados indican (Llorens et al., 2003b) que respecto a la escorrentía en condiciones actuales, es decir con los porcentajes de cubiertas actuales en Can Vila ( $70 \%$ de prados y $30 \%$ de pinares), si se produjera una reforestación total de la cuenca, los caudales disminuirían en un $18 \%$, y en el caso de que el cambio fuese a una cuenca totalmente cubierta por robles la disminución sería un poco menor (14\%). El aumento de la interceptación por una cuenca totalmente cubierta por coníferas se ve ligeramente compensado por una reducción de la transpiración, respecto a los prados, pero esta reducción no es suficiente para compensar la disminución del caudal. En el caso de las caducifolias la reducción del caudal es menos extrema debido también a una menor interceptación.

\section{Conclusiones}

Durante parte del año, las cuencas de Vallcebre funcionan como cuencas húmedas, la interceptación de la precipitación está determinada por la duración de los eventos, no se produce una reducción importante de la transpiración de prados y árboles por déficit hídrico, y los flujos superficiales alimentan tanto las áreas saturadas como el caudal de base. El aumento de la demanda evapotranspirativa durante el verano produce una reducción de la reserva hídrica. La limitación del agua disponible en el suelo provoca una restricción importante de las tasa de transpiración de la vegetación y una interrupción de la recarga. Paralelamente durante el verano, los procesos de interceptación son más complejos debido a una combinación mayor de factores determinantes, es decir una mayor variabilidad de las intensidades de precipitación y de las condiciones de demanda atmosférica. 
A esta variabilidad estacional de los procesos hidrológicos, en el ambiente de montaña Mediterránea estudiado, se le sobrepone una importante complejidad espacial, concretada en las diferentes cubiertas vegetales presentes (prados, "manchas" de pinares o de robledales), que determina tanto los procesos hídricos dominantes a escala de cubierta como el balance hídrico local. Influenciando estos significativamente los procesos y el balance hidrológico a escala de cuenca.

\section{Agradecimientos}

Estas investigaciones han sido financiadas por el acuerdo entre el CSIC y el Ministerio de Medio ambiente (Red RESEL del proyecto LUCDEME), así como por los proyectos: EPOHIDRO (HID98-1056-C02-01), PROHISEM (REN2001-2268-C0101/HID), PIRIHEROS (REN2003-08678/HID) y CANOA (CGL2004-04919-C02-01) financiados por el Plan Nacional de Investigación Científica y Técnica; y VAHMPIRE (ENV4-CT95-0134) y TEMPQSIM (EVK1-CT-2002-00112) financiados por la comisión europea.

El segundo autor disfruta de una beca predoctoral FPI del MCYT-MEC, el tercer autor de un contrato postdoctoral I3P del CSIC y el cuarto de una beca predoctoral FPU del MEC.

Los autores agradecen la colaboración de M. Abril, O. Avila, O. Cornellà, J. Delgado, N. Martínez, I. Oliveras, X. Huguet, R. Poch, D. Rabadà, D. Regüés, C. Salvany y M. Soler.

\section{Referencias bibliográficas}

Balasch, J.C., CAstelltort, F.X., Llorens, P. y Gallart, F. (1992). Hydrological and sediment dynamics network design in a Mediterranean mountainous area subject to gully erosion. En: Bogen, J., Walling, D.E., Day, T. (Eds.), Erosion and sediment transport monitoring programmes in river basins. IAHS Pub., 210: 433-442.

Bosch, J. M. y HewletT, J. D. (1982). A review of catchment experiments to determine the effect of vegetation changes on water yield and evapotranspiration. Journal of Hydrology, 55: 3-23.

Cermák J., Nadezhdina, N., Raschi, A. y Tognetti, R. (1998). Sap flow in Quercus pubescens and Quercus cerris stands in Italy. En: International Workshop on Measuring Sap Flow in Intact plants, Zidlochovice, Czech Republic. IUFRO Publications. Mendel University. 
Gallart, F., Llorens, P., LATRON, J. y REGÜÉS, D. (2002). Hydrological processes and their seasonal controls in a small Mediterranean mountain catchment in the Pyrenees. Hydrology and Earth System Sciences, 6(3): 527-537.

GAllart, F. y LlORENS, P. (2004). Observations on land cover changes and water resources in the headwaters of the Ebro catchment, Iberian Peninsula. Physics and Chemistry of the Earth, parts A/B/C (11-12): 769-773.

Gallart, F., Balasch, C., Regüés, D., Soler, M. y Castelltort, X. (2005a). Catchment dynamics in a Mediterranean mountain environment: the Vallcebre research basins (South Eastern Pyrenees). II Erosion and sediment dynamics. En: C. García and R. Batalla (Eds.), Catchment dynamics and river processes: latest research with examples from the Mediterranean climate regions. Elsevier, 17-29.

GAllart, F., LATRON, J., Llorens, P. (2005b). Catchment dynamics in a Mediterranean mountain environment. The Vallcebre research basins (South Eastern Pyrenees) I: Hydrology. En: C. García and R. Batalla (Eds.), Catchment dynamics and river processes: latest research with examples from the Mediterranean climate regions. Elsevier, 1-16.

Gallart, F., Llorens, P., LATron, J., RegüÉs, D. y Soler, M. (En prensa). Procesos hidrológicos y dinámica de sedimentos en áreas de montaña subhúmeda Mediterránea (Vallcebre). En: La Red de Estaciones experimentales de Seguimiento y Evaluación de la Desertificación. Ministerio de Medio Ambiente.

García-Ruiz, J. M., Lasanta, T., Ruiz-Flaño, P., Ortigosa, L., White, S., GONZÁLEZ, C. y MARTí, C. (1996). Land-use changes and sustainable development in mountain areas: a case study in the Spanish Pyrenees. Landscape Ecology, 11 (5): $267-277$.

GASH, J.H.C. (1979). An analytical model of rainfall interception by forest. Quarterly Journal Royal Meteorological Society, 105(443): 43-55.

GRANIER, A. (1985). Une nouvelle méthode pour la mesure du flux de sève brute dans le tronc des arbres. Annales des Sciences Forestieres, 42(2): 193-200.

GRANIER, A. (1987). Evaluation of transpiration in a Douglas-fir stand by means of sap flow measurements. Tree physiology, 3: 309-320.

GRANIER, A. y Loustaud, D. (1994). Measuring and modelling the transpiration of a maritime pine Canopy for sap-flow data. Agricultural and Forest Meteorology, 71: 6181.

LATRON, J. (2003). Estudio del funcionamiento hidrológico de una cuenca mediterránea de montaña (Vallcebre, Pirineos Catalanes). Ph.D. Thesis. Facultat de Geologia, Universitat de Barcelona, 269 pp.

LlORENS, P. (1991). Resposta hidrològica i dinàmica de sediments en una petita conca pertorbada de muntanya Mediterrània, Ph.D. Thesis, Facultat de Geografia i 
Història, Universitat de Barcelona, 276 pp.

LlORENS, P. (1993). Hidrological implications of afforestation of abandoned lands: water balance simulation of a small Mediterranean basin. Acta Geologica Hispanica, 28 (2-3): 131-138.

LLORENS, P. (1997). Rainfall interception by a Pinus sylvestris forest patch overgrown in a Mediterranean mountainous abandoned area II. Assessment of the applicability of Gash's analytical model. Journal of Hydrology, 199: 346-359.

LlORENS, P. y GALLART, F. (1992). Small basin response in a Mediterranean mountainous abandoned farming area: research design and preliminary results. Catena, 19: 309-320.

Llorens, P., Poch, R., Latron, J. y Gallart, F. (1997). Rainfall interception by a Pinus sylvestris forest patch overgrown in a Mediterranean mountainous abandoned area. I. Monitoring design and resu lts down to the event scale. Journal of Hydrology, 199: 331-345.

LlORENS, P. y GALLART, F. (2000). A simplified method for forest water storage capacity measurement. Journal of Hydrology, 240: 131-144

Llorens, P., LATRON, J. y GALlART, F. (2003a). Dinámica espacio-temporal de la humedad del suelo en un área de montaña mediterránea. cuencas experimentales de Vallcebre (Alto Llobregat). En: Álvarez-Benedí y P. Marinero (Eds): Estudios de la Zona No Saturada del Suelo, Vol. VI.: 71-76.

Llorens, P., Latron, J. y Oliveras, I. (2003b). Modelización del efecto del cambio global en la hidrología superficial. Ejemplo de aplicación a una cuenca Mediterránea de montaña. $3^{a}$ Asamblea Hispano-Portuguesa de Geodesia y Geofísica. Proceedings Tomo III: $1679-1681$.

Llorens, P., OliVeras, I. y POYATOS. R. (2003c). Temporal variability of water fluxes in a Pinus sylvestris forest patch in Mediterranean mountain conditions. (Vallcebre research catchments, Catalan Pyrenees). IAHS Pub. 278: 101-105.

NASH, I.E. y SutclifFE, I.V. (1970). River flow forescasting through conceptual models. Journal of Hydrology, 10: 282-290.

OliVeras, I. y LLORENS, P. (2001). Medium-term sap flux monitoring in a Scots pine stand: analysis of the operability of the heat dissipation method for hydrological purposes. Tree Physiology, 21: 473-480.

Oren, R., Sperry, J.S., Katul, G.G., Pataki, D.E., Ewers, B.E., Phillips, N. y SCHAFER, K.V.R. (1999). Survey and synthesis of intra-and interspecific variation in stomatal sensitivity to vapour pressure deficit. Plant Cell and Environment, 22(12): 1515-1526.

POyATOS, R., LATRON, J. y LlORENS, P. (2003). Land-Use and Land-Cover Change After Agricultural Abandonment. The Case of a Mediterranean Mountain Area (Catalan 
Pre-Pyrenees). Mountain Research and Development, 23 (4): 52-58.

POyATOS, R. y LlORENS, P. (2003). Influencia de la humedad del suelo y del período de actividad vegetal en la evapotranspiración de un pastizal montano mesófilo. Actas del VII Congreso Nacional de la Asociación Española De Ecología Terrestre, 182-196.

Poyatos, R., Llorens, P. y Gallart, F. (2005). Transpiration of montane Pinus sylvestris L. and Quercus pubescens Willd. Forest stands measured with sap flow sensors in NE Spain. Hydrology and Earth Systems, 9: 493-505.

RABADÀ, D. (1995). Dinàmica hidrològica d'una petita conca pirenaica de camps abandonats amb pinedes en expansió (Alt Berguedà, Barcelona). Ph.D. Thesis. Facultat de Geologia. Universitat de Barcelona. 321 pp.

Rubio, C. y LloREns, P. (2003). Uso de funciones de edafotransferencia para la determinación de propiedades hidrodinámicas de los suelos en una cuenca experimental (Vallcebre, Alt Llobregat). En: Álvarez-Benedí, J. y Marinero, P. (Eds.), Estudios de la Zona No Saturada del Suelo. Vol. VI: 137-142.

Rubio, C. y Llorens, P. (2004). Comparing different pedotransfer functions for silty loam soils in a Mediterranean mountain catchment, En: Aagaard, P.; Bedbur, E.; Bidoglio, G.; Candela, L.; Nuetzmann, G.;Trevisan, M.;Vanclooster, M. y Viotti, P.(Eds.), Saturated and unsaturated zone: integration of process knowledge into effective models, Roma: $480 \mathrm{p}$.

SAHIN, V. y HALL, M. J. (1996). The effects of afforestation and deforestation on water yields. Journal of Hydrology, 178: 293-309.

SchaAp, M.G., LeIJ, F.J. y VAn GENUChTEN, M. TH. (2001). ROSETTA: a computer program for estimating soil hydraulic parameters with hierarchical pedotransfer functions. Journal of Hydrology, 251: 163-176.

Sivapalan, M., Takeuchi, K., Franks, S.W., Gupta, V.K., Karambiri, H., Lakshmi, V., Liang, X., Mcdonnell, J.J., Mendiondo, E.M., O’Connell, P.E., OKi, T., Pomeroy, J.W., Schertzer, D., Uhlenbrook, S. y ZeHE, E. (2003). IAHS Decade on Predictions in Ungauged Basins (PUB), 2003-2012: Shaping an exciting future for the hydrological sciences. Hydrological Sciences Journal, 48(6): 857.

TOEBES, C. y OURYVAEV, V. (Eds.) (1970). Les bassins répresentatifs et expérimentaux, Guide international des pratiques en matière de recherche. UNESCO, Paris, France, 380pp.

Vachaud G., Passerat de Silane A., Balabanis P. y Vauclin M. (1985). Temporal stability of spatially measured soil water probability density function. Soil Science Society American Journal, 49: 822-827. 